\title{
The role of nitric oxide in the dysregulation of the urine concentration mechanism in diabetes mellitus
}

\section{Penelope Cipriani, Sunhye L. Kim, Janet D. Klein, Jae H. Sim, Tobias N. von Bergen and Mitsi A. Blount*}

Renal Division, Department of Medicine, Emory University, Atlanta, GA, USA

Edited by:

Michael B. Butterworth, University of

Pittsburgh School of Medicine, USA

\section{Reviewed by:}

Pablo A. Ortiz, Wayne State

University, USA

Oleh Pochynyuk, University of Texas

Health Science Center - Houston,

USA

${ }^{*}$ Correspondence:

Mitsi A. Blount, Renal Division,

Department of Medicine, Emory

University, 338D Woodruff Memorial

Building, 1639 Pierce Drive, Atlanta,

GA 30322, USA.

e-mail:mabloun@emory.edu
Uncontrolled diabetes mellitus results in osmotic diuresis. Diabetic patients have lowered nitric oxide (NO) which may exacerbate polyuria. We examined how lack of NO affects the transporters involved in urine concentration in diabetic animals. Diabetes was induced in rats by streptozotocin. Control and diabetic rats were given L-NAME for 3 weeks. Urine osmolality, urine output, and expression of urea and water transporters and the $\mathrm{Na}-\mathrm{K}-2 \mathrm{Cl}$ cotransporter were examined. Predictably, diabetic rats presented with polyuria (increased urine volume and decreased urine osmolality). Although metabolic parameters of control rats were unaffected by L-NAME, treated diabetic rats produced $30 \%$ less urine and osmolality was restored. UT-A1 and UT-A3 were significantly increased in diabetic rat inner medulla. While L-NAME treatment alone did not alter UT-A1 or UT-A3 abundance, absence of NO prevented the upregulation of both transporters in diabetic rats. Similarly, AQP2 and NKCC2 abundance was increased in diabetic animals however, expression of these transporters were unchanged by L-NAME treatment of diabetes. Increased expression of the concentrating transporters observed in diabetic rats provides a compensatory mechanism to decrease solute loss despite persistent glycosuria. Our studies found that although diabetic-induced glycosylation remained increased, total protein expression was decreased to control levels in diabetic rats treated with L-NAME. While the role of NO in urine concentration remains unclear, lowered $\mathrm{NO}$ associated with diabetes may be deleterious to the transporters' response to the subsequent osmotic diuresis.

Keywords: diabetes mellitus, urine concentration, osmotic diuresis, urea transporter

\section{INTRODUCTION}

Diabetes mellitus (DM) is one of the leading causes of death in the United States and is the most common cause of end-stage renal disease (Centers for Disease Control and Prevention, 2008). Advancing diabetes results in osmotic diuresis and polyuria placing the patient at risk for hypovolemic shock. Urea transporters, UT-A1 and UT-A3, and the $\mathrm{Na}^{+}-\mathrm{K}^{+}-2 \mathrm{Cl}^{-}$cotransporter (NKCC2) are solute transporters that are not only critical for urine concentration but also control tubule osmotic pressure via regulation of urea and sodium levels. In the inner medulla (IM), the vasopressinsensitive water channel aquaporin 2 (AQP2) is an important contributor to water reabsorption. The ensuing osmotic diuresis and polyuria that occurs with advancing diabetes makes these transporters of particular interest for our studies.

Evidence suggests that the decline in renal function associated with advancing diabetes is due to a prolonged state of nitric oxide (NO) deficiency (Huang et al., 2009). NO is generated from nitric oxide synthase (NOS). There are three distinct isoforms of this enzyme, all of which are expressed in the kidney: NOS1 (neuronal or nNOS), NOS2 (inducible or iNOS), and NOS3 (endothelial NOS or eNOS; Wu et al., 1999). Patients with deleterious polymorphisms of NOS3 are more susceptible to developing diabetic nephropathy (He et al., 2011); an effect that was confirmed in NOS3 deficient mice with diabetes (Zhao et al., 2006). The decline in NOS activity in the renal medulla is not however, altered by glucose-dependent osmotic diuresis alone (Lee et al., 2005).
This does not exclude NO involvement in the urine concentration mechanism. In fact, the IM, which is the primary site of urine concentration, expresses all three isoforms of NOS and has the highest capacity for NO synthesis compared to other nephron segments (Wu et al., 1999). Similar to uncontrolled DM, transgenic mice with all three NOS isoforms ablated also display polyuria (Morishita et al., 2005).

Although not extensive, there is increasing data investigating the effect of $\mathrm{NO}$ on the transporters involved in urine concentration. NO stimulates a cGMP-mediated pathway that results in phosphorylation and trafficking of AQP2 to the apical plasma membrane of the inner medullary collecting duct (IMCD) where the transporter is functional (Bouley et al., 2005). NO inhibits NKCC2, hindering sodium transport in the thick ascending limb (Herrera et al., 2009). Urea transport does not appear to be affected by either cGMP or NO (Nonoguchi et al., 1988). Rather, inhibition of NO production increases concentrations of superoxide $\left(\mathrm{O}_{2}^{-}\right)$which results in increased urea transport in the IMCD (Zimpelmann et al., 2003).

Declining NO concentration in the diabetic kidney may exacerbate the potential for hypovolemic shock by further disturbing the urine concentration mechanism, which is already compromised by osmotic diuresis. Therefore, the present study was designed to investigate if the diabetes-driven compensatory action of various transporters involved in concentrated 
urine production is compromised in the absence of $\mathrm{NO}$ production.

\section{MATERIALS AND METHODS ANIMALS}

Animal protocols were approved by the Emory University Institutional Animal Care and Use Committee. Male Sprague Dawley rats weighing 150-200 g, were allowed free access to water and fed standard rat chow. Rats were injected (tail-vein) with streptozotocin $(62.5 \mathrm{mg} / \mathrm{kg}$ ) to induce DM, as reported previously (Blount et al., 2008). Hyperglycemia was verified $24-48 \mathrm{~h}$ after injection using a Lifescan Ultra II glucometer. Control and DM rats were given L-NAME $(50 \mathrm{mg} / \mathrm{kg} /$ day $)$ via $5 \mathrm{ml}$ of drinking water for 3 weeks following the 4-days after STZ injection. After the rat consumed the full dose of L-NAME, the regular water bottle was provided for ad libitum water for the remainder of that 24 -h period. This was repeated each day.

\section{METABOLIC MEASUREMENTS}

Rats from all four experimental groups were placed in metabolic cages for $24 \mathrm{~h}$ before sacrifice and urine was collected under oil to prevent evaporation. Urine osmolality was measured on Wescor 5520 Vapor Pressure Osmometer (Wescor). Urine urea concentration was determined using Infinity Urea Reagent from Thermo Scientific (Thermo Fisher Scientific). Urinary nitrate/nitrite (NOx) levels were measured in urine as nitric oxide metabolites using a Cayman Fluorometric Assay. Blood glucose was determined before sacrifice with the glucometer.

\section{SAMPLE PREPARATION AND WESTERN BLOT ANALYSIS}

Kidneys were removed and dissected into outer medulla (OM), base of the IM, and tip of the IM. Tissues were placed into ice-cold isolation buffer $(10 \mathrm{mM}$ triethanolamine, $250 \mathrm{mM}$ sucrose, $\mathrm{pH}$ 7.6, $1 \mu \mathrm{g} / \mathrm{ml}$ leupeptin, and $40 \mu \mathrm{g} / \mathrm{ml}$ PMSF) and homogenized with glass homogenizers. SDS was added to a final concentration of $1 \%$, and the samples were sheared with a 25 -gage needle. Homogenates were centrifuged at $8,000 \mathrm{~g}$ for $15 \mathrm{~min}$, and the protein in the supernatant fractions was measured by a modified Lowry method (DC Protein Assay Kit; Bio-Rad). For western blot analysis, proteins (10-20 $\mu \mathrm{g} / \mathrm{lane})$ were first size separated by SDS-PAGE and then electroblotted to polyvinylidene difluoride membranes (Millipore). Membranes were probed with primary antibody overnight at $4^{\circ} \mathrm{C}$. Antibodies that were derived and characterized in this laboratory include a COOH-terminal UT-A1 (detects UT-A1 exclusively), $\mathrm{NH}_{2}$-terminal UT-A1 (detects UTA1 and UT-A3 simultaneously), AQP2, and NKCC2 were used to determine the level of respective protein abundances (Blount et al., 2008). The secondary antibody used for detection was Alexa Fluor 680-linked anti-rabbit IgG (Invitrogen). Signal was detected with a LI-COR system. Using LI-COR Odyssey software, densitometry was determined for each protein. Results reflect the ratio of the densitometry of the detected protein to the densitometry of $\beta$ actin where $\beta$ actin (antibody from Sigma Aldrich) served as a loading control.

\section{STATISTICAL ANALYSIS}

Values are SE from each experimental group where $n=5$. Densitometry ratios were calculated based on $\beta$ actin loading controls. The densitometries from each group of animals were averaged and the data were presented as means \pm SE for the percent change from the control value. To test for statistical significance between the multiple groups, we used an ANOVA followed by Newman-Keuls test. The criterion for statistical significance was $p<0.05$.

\section{RESULTS}

\section{ADMINISTRATION OF L-NAME ALTERS METABOLIC PROGRESSION OF} DIABETES

Age-matched male Sprague Dawley rats from the following experimental groups were individually placed in metabolic cages to monitor physiological changes for $24 \mathrm{~h}$ : (1) control rats, (2) rats treated with L-NAME, (3) DM rats, and (4) DM rats treated with L-NAME (Table 1). DM animals showed elevated blood glucose at the time of sacrifice confirming hyperglycemia. L-NAME did not change blood glucose levels of either control or DM animals. DM rats had a higher urine output and lower urine osmolality than control animals. L-NAME-treated animals did not have a significant increase in urine volume compared to control animals however, urine osmolality was significantly decreased. L-NAME treatment of DM animals decreased urine output compared to untreated DM rats. These animals still produced a greater volume of urine than the control animals. Although urine osmolality of L-NAME-treated DM animals was higher than untreated DM rats, the reported value did not reach significance. Rats treated with L-NAME had no change in urine urea however DM rats had a significantly lower urine urea corresponding with the osmolality decrease. L-NAME treatment of DM rats slightly increased urine urea. While this change was not significant from DM values, the amount of urine urea was not different than control rats. To reflex the amount of nitric oxide excreted in the urine, we measured urinary nitrate/nitrite (NOx) levels. NOx levels were lowered $68 \%$ in rats treated with L-NAME when compared to control animals, confirming that L-NAME was inhibiting the production of nitric oxide. Diabetic rats also had lowered urine NOx levels (77\%) compared to control rats. This is not surprising given that $\mathrm{NO}$ concentration is lower in kidneys of STZ-injected diabetic rats (Palm et al., 2005). L-NAME treatment of DM rats reduced the already lowered levels of NOx in the urine slightly.

\section{ADMINISTRATION OF L-NAME REDUCED THE COMPENSATORY UPREGULATION OF UREA TRANSPORTERS NORMALLY OBSERVED WITH DM}

UT-A1, a glycoprotein, is expressed in both the papilla and IM base. Although the functional difference remains a mystery, abundance of the two glycoproteins of UT-A1 (117 and 97-kDa) differ based on tissue location. Western analysis of control rats detected both glyco-forms of UT-A1 in the IM tip (Figure 1A) and the predominant $97-\mathrm{kDa}$ form in IM base (Figure 1D). L-NAME treatment alone did not change the total protein abundance of UT-A1 in either section of the IM nor did the inhibition of NO change the glycosylation state (Figure 1). UT-A1 expression was significantly upregulated in both the IM tip and base of diabetic rats. This was largely attributable to increased expression of the $117-\mathrm{kDa}$ form (Figures 1C,F). Diabetic rats treated with L-NAME did not have a significant increase in total UT-Al protein abundance (97- or $117-\mathrm{kDa})$ in the IM tip (Figures $\mathbf{1 B}, \mathbf{C}$ ). In the IM 
Table 1 | Effects of L-NAME administration on control and diabetic rats.

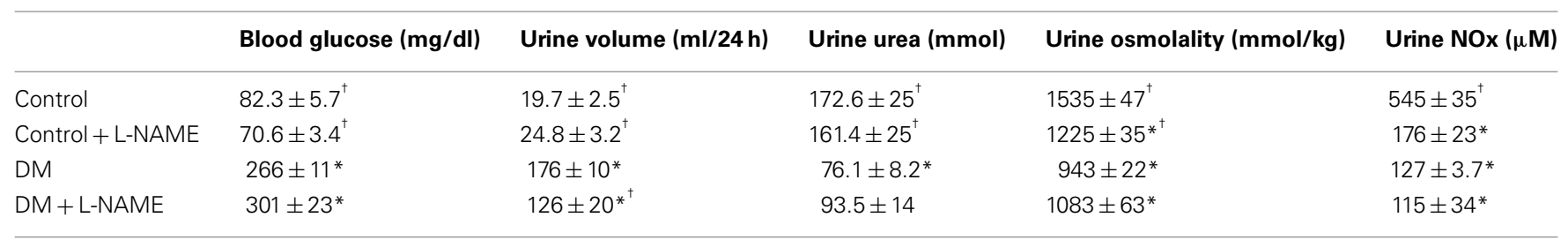

${ }^{*} p>0.05$ compared to control.

${ }^{\dagger} p>0.05$ compared to DM.

Newman-Keuls test.

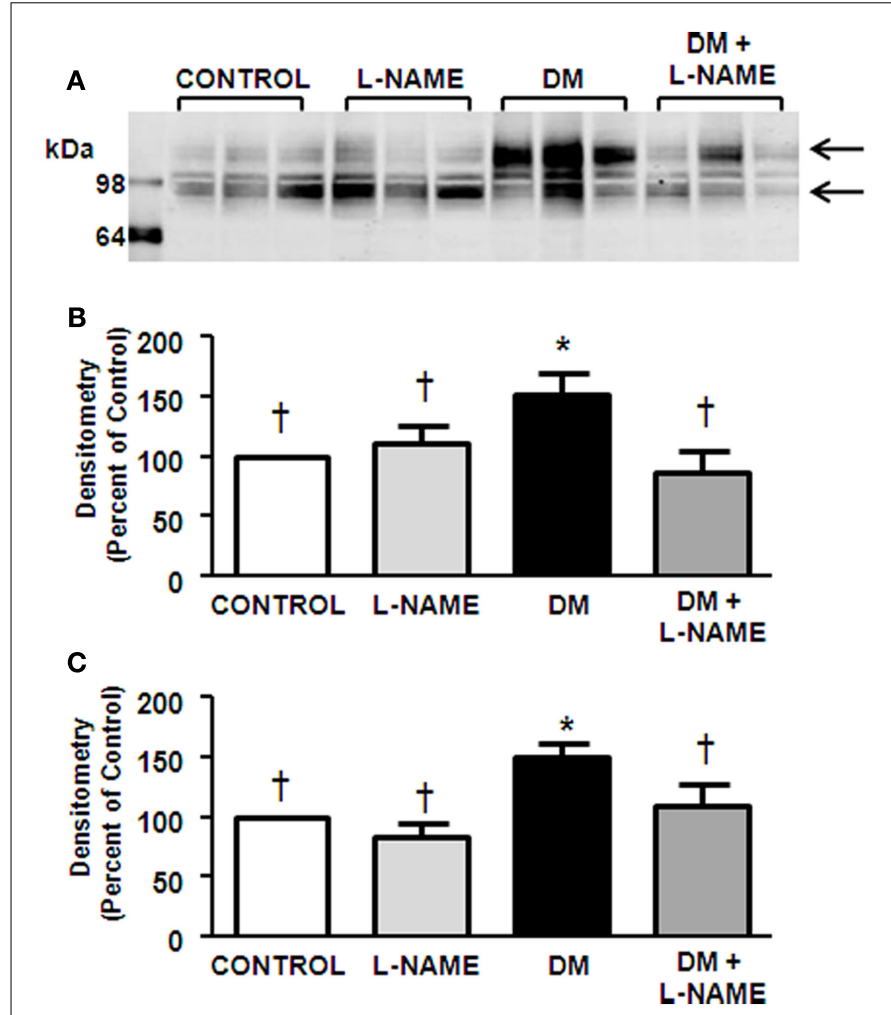

FIGURE 1 | Upregulation of UT-A1 in diabetes is blunted by NO inhibition. Shown is a representative western blot of inner medulla (IM) tip (A) and base (D) probed for UT-A1 where each lane represents one rat. Densitometry was determined for the $97-k D a(B)$ and $117-k D a$ glyco-forms (C) in IM tip and the
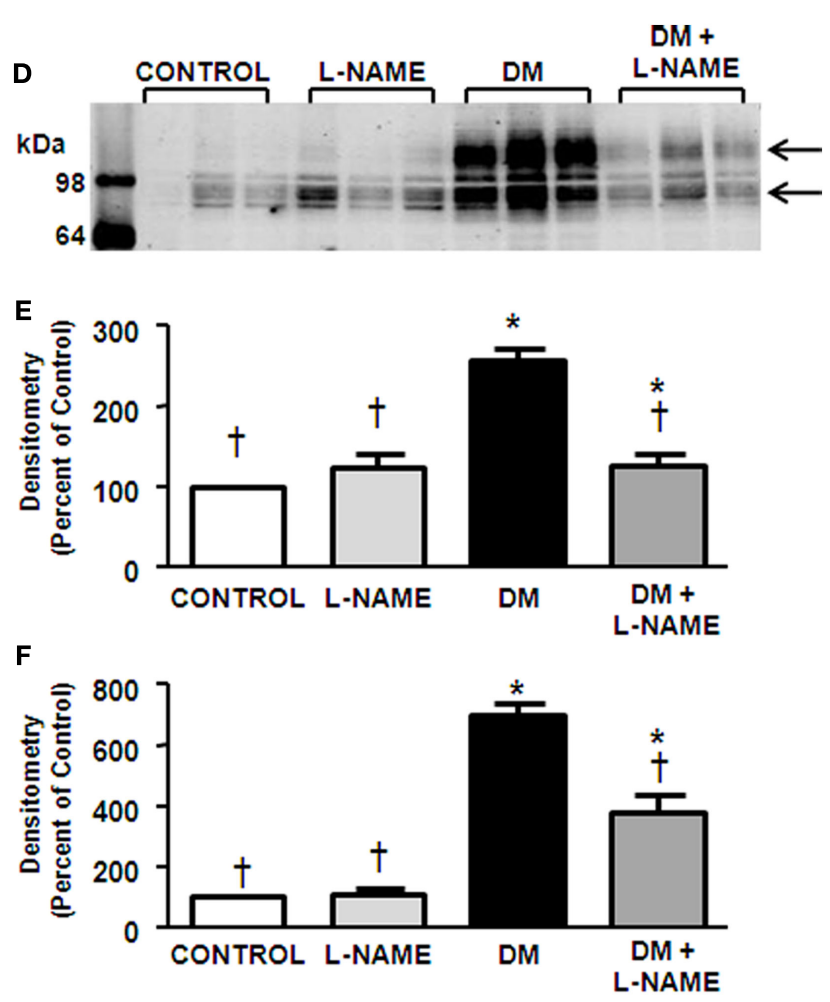

97-kDa (E) and 117-kDa glyco-forms (F) in IM base. The experimental conditions were performed 5 times $(n=5)$ where there were 5 animals per experimental group in each cohort. In total, 25 animals per experimental group were analyzed. ${ }^{*} p<0.05$ compared to control, ${ }^{\dagger} p<0.05$ compared to DM. base of L-NAME-treated diabetic animals, 97-kDa UT-A1 abundance was statistically increased when compared to control rats but expression of this glycoprotein was also significantly decreased compared to diabetic rats (Figure 1E). L-NAME treatment of diabetic animals did inhibit induction of the $117-\mathrm{kDa}$ form of UT-A1 however the amount of $117-\mathrm{kDa}$ protein is $\sim 3.5$-fold over basal levels (Figure 1F).

UT-A3 is also a glycoprotein that is found predominantly in the papilla in the IM. This transporter is distinguished as multiple glyco-forms ranging from 45 - to $65-\mathrm{kDa}$ (Blount et al., 2008) as detected in control rats (Figure 2A). Rats treated with L-NAME did not demonstrate a change in UT-A3 glycoprotein abundance (Figures 2B,C). Corroborating previous reports (Blount et al.,
2008), UT-A3 abundance was increased in DM rats (Figure 2B). The increased protein abundance of UT-A3 is due to increased glycosylation of the upper form of the protein (Figure 2B) whereas induction of diabetes had no effect on the 45-kDa UT-A3 (Figure 2C). L-NAME treatment of the DM rats prevented the compensatory increase in UT-A3 abundance (Figure 2). Interestingly, L-NAME treatment of diabetic rats significantly reduced the 45-kDa glycosylated form of UT-A3 (Figure 2C).

\section{L-NAME REDUCED THE DM-INDUCED UPREGULATION OF THE WATER CHANNEL, AQP2}

AQP2 is expressed as glycosylated (40-46-kDa) and unglycosylated (29-kDa) proteins (Nejsum et al., 2001) as observed in control 

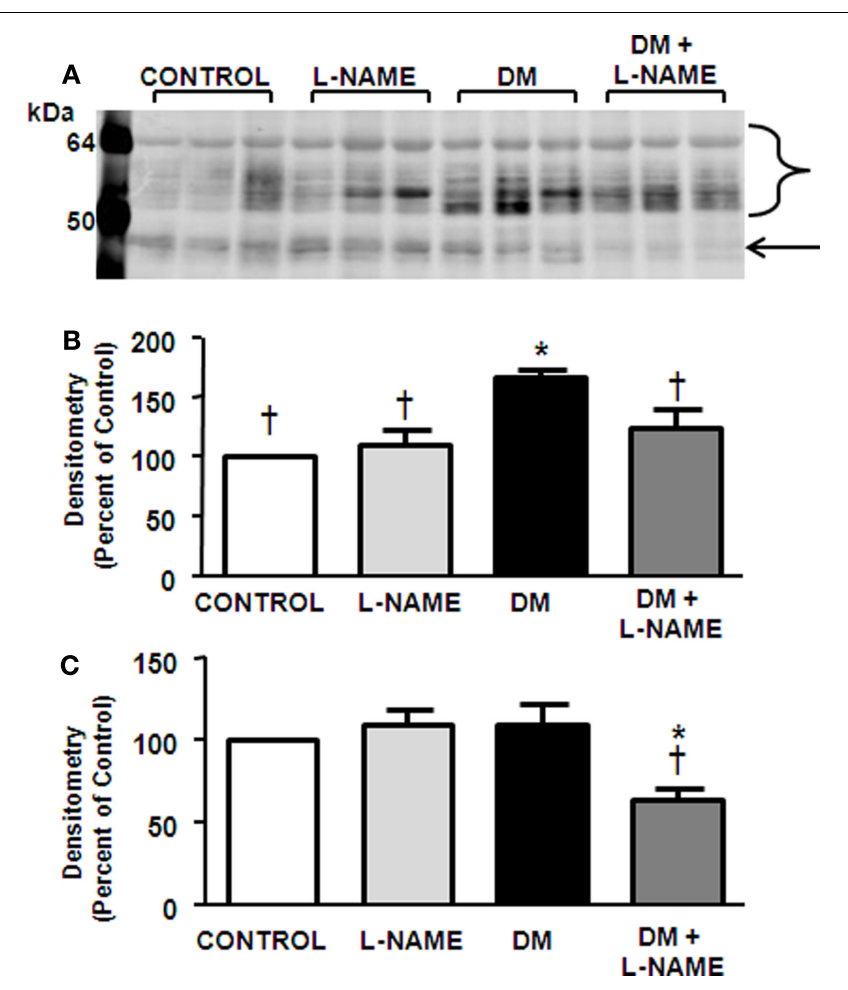

FIGURE 2 | Upregulation of UT-A3 in diabetes is blunted by NO inhibition. Presented is a representative western blot of IM tip (A) probed for UT-A3. Densitometry was determined for all the 65-kDa smear (B) and 45-kDa glyco-form (C) in the IM tip. The experimental conditions were performed five times $(n=5)$ where there were five animals per experimental group in each cohort. In total, 25 animals per experimental group were analyzed. ${ }^{*} p<0.05$ compared to control, ${ }^{\dagger} p<0.05$ compared to DM.

rat IM tip and base (Figures 3A,D). L-NAME treatment did not affect AQP2 expression in the papilla but did lower protein levels in the IM base (Figures 3B,E). Glycosylated AQP2 protein levels were significantly increased in both the tip and base of diabetic IM (Figures 3B,E); however, the unglycosylated form of AQP2 was not altered in response to diabetes in either IM tip or base (Figures 3C,F). In the IM tip of diabetic animals, L-NAME treatment had no effect on the unglycosylated AQP2 expression (Figure 3C) but did reduce the glycosylated AQP2 abundance to basal level (Figure 3B). L-NAME treatment of DM animals lowered glycosylated AQP2 levels in the IM base compared to DM animals (Figure 3E) but did not alter unglycosylated AQP2 abundance (Figure 3F).

\section{NKCC2 WAS INCREASED IN DM ANIMALS BUT EXPRESSION LEVELS WERE UNAFFECTED BY L-NAME}

NKCC2 was detected at $150-\mathrm{kDa}$ in the OM dissected from rat kidney (Figure 4A). Rats treated with L-NAME did not have any alteration in NKCC2 protein abundance (Figure 4B). STZ-treatment significantly increased NKCC2 levels (Figure 4B). L-NAME treatment did not significantly change the increased NKCC2 abundance in DM rats.

\section{DISCUSSION}

The goal of this study was to determine how declining $\mathrm{NO}$ in the diabetic kidney affects the already compromised urine concentrating mechanism. Using STZ-induced diabetic rats, we found that L-NAME-mediated inhibition of NO alleviated the polyuria observed in untreated diabetes. L-NAME treatment alone did not alter basal levels of blood or urine glucose suggesting that L-NAME treatment of the DM kidney does not improve polyuria by altering glucose-dependent osmotic diuresis but by other mechanisms, including differential expression of the concentrating transporters UT-A1, UT-A3, and AQP2.

The effect of untreated DM on urea transporter expression and function in the IM has been extensively investigated (Kim et al., 2003, 2005; Blount et al., 2008). Collectively, these studies have found that DM induces an increase in both UT-A1 and UT-A3 protein abundance in an attempt to restore inner medullary interstitial urea, which is disrupted in the advancement of the disease. Our results present the novel finding that diabetic rats treated with L-NAME did not have the compensatory increase in UTA1 or UT-A3 expression. Previous work has shown that UT-A1 protein abundance increases during osmotic diuresis whenever urinary urea decreases in order to continuously transport urea to the interstitium (Kim et al., 2005). Although DM resulted in decreased urea as a urinary solute in this study, L-NAME treatment of DM rats increased the amount of urea in urine, possibly explaining the dampened increase of urea transporter proteins in the face of DM.

Early work demonstrated that both glycoproteins of UT-A1 are equally upregulated in the papilla in response to chronic diabetes; however, increased protein expression of UT-A1 in the IM base of diabetic rats was mainly due to an increase in the 117$\mathrm{kDa}$ form (Kim et al., 2003). Our findings are in agreement; total UT-A1 expression was significantly upregulated throughout the IM of diabetic rats, particularly in the IM base. Despite the decrease in overall protein expression, L-NAME-treated DM animals still had an increase in the $117-\mathrm{kDa}$ glycoprotein. Recently, Chen et al. (2011) determined that the 117-kDa UT-A1 was the mature glycosylation form of the transporter. Furthermore, the researchers found that the $117-\mathrm{kDa}$ form is associated with lipid rafts. This localization of $117-\mathrm{kDa}$ UT-A1 into lipid rafts was increased in response to diabetes. Although not proven to be the mature glycosylation form of UT-A3, we found that uncontrolled diabetes increased the upper $65-\mathrm{kDa}$ glycosylated form while the lower, $45-\mathrm{kDa}$ form remained unchanged. Hyperglycemia has been linked to increased glycosylation of a variety of proteins (Martin et al., 2006). It is possible that uncontrolled diabetes increases UT-A1 and UT-A3 membrane trafficking by changing the glycosylation state, which facilitates lipid raft targeting; however, we did not examine altered cellular location in our studies. Although hyperglycemia-induced glycosylation may account for the increased presence of the $117-\mathrm{kDa}$ form of UT-A1 and $65-\mathrm{kDa}$ form of UT-A3 independent of nitric oxide, it does not explain the dampened total protein abundance. Further studies addressing the function of the urea transporter glycosylation states and lipid rafts in diabetes will need to be pursued to completely elucidate this question. 


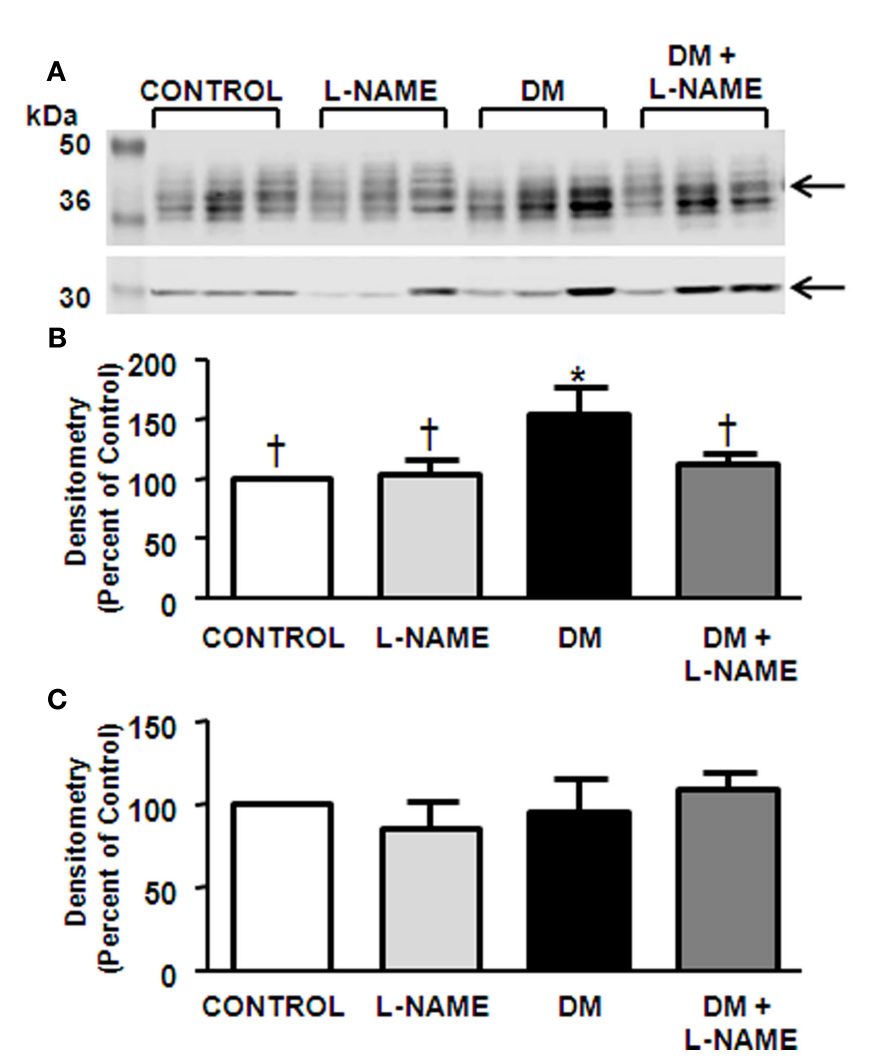

FIGURE 3 | Upregulation of glycosylated AQP2 in diabetes is blunted by NO inhibition. Displayed is a representative western blot of IM tip (A) and base (D) probed for AQP2. Densitometry was determined for the

glycosylated (B) and unglycosylated forms (C) in the IM tip as well as the glycosylated (E) and unglycosylated forms (F) in the IM base. To prevent saturation of bands, the blot was scanned at a lighter intensity to measure

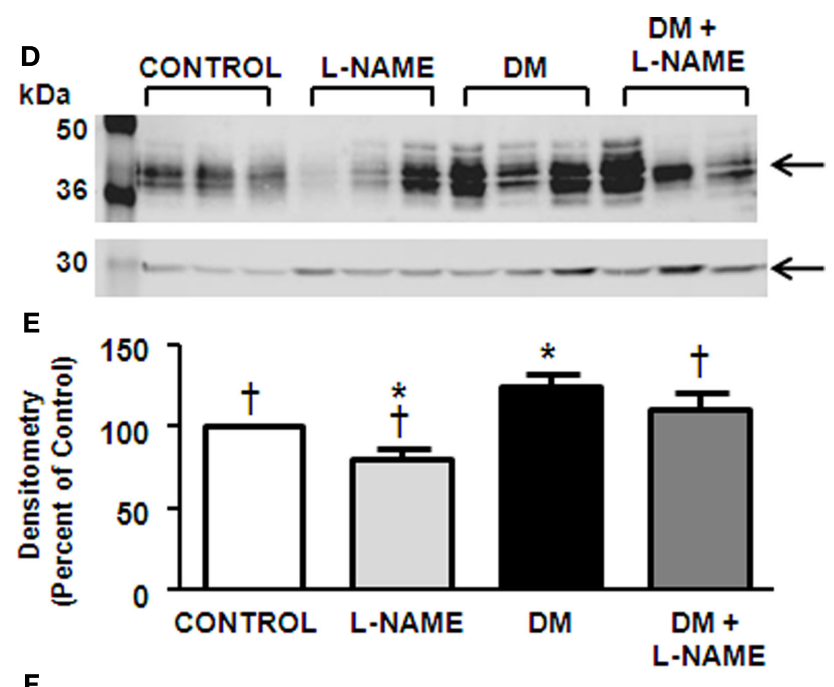

$\mathbf{F}$

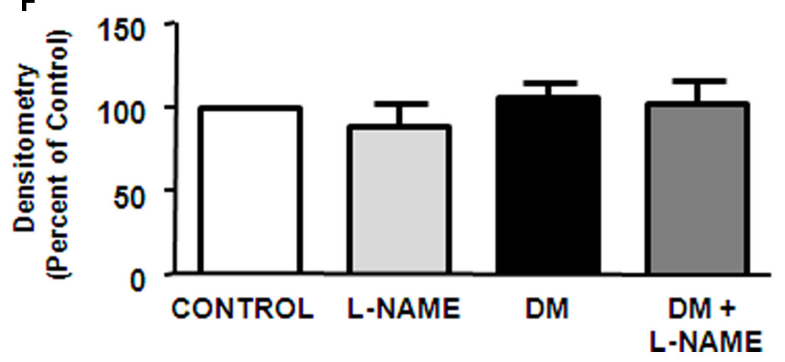

glycosylated AQP2 (A,D) and at a higher intensity to measure the unglycosylated AQP2 (A,D) however, images are gleaned from the same representative western blot. The experimental conditions were performed five times $(n=5)$ where there were five animals per experimental group in each cohort. In total, 25 animals per experimental group were analyzed. ${ }^{*} p<0.05$ compared to control, ${ }^{\dagger} p<0.05$ compared to DM.
Investigation of AQP2 revealed that L-NAME treatment did not affect AQP2 expression in the papilla but did lower protein levels in the IM base. This corresponds to studies that documented decreased AQP2 expression in the IM of rats orally treated with L-NAME for 6 weeks (Albertoni Borghese et al., 2007). We found that glycosylated AQP2, but not unglycosylated AQP2, is upregulated in response to diabetes which is in agreement with previously published reports (Nejsum et al., 2001; Satake et al., 2010). Although not investigated in this study, uncontrolled diabetes can increase vasopressin plasma levels (Trinder et al., 1994; Iwasaki et al., 1996; Bardoux et al., 1999). This could explain the increase in AQP2 expression in response to diabetes in that vasopressin can upregulate AQP2 protein levels at a transcriptional level (Nielsen et al., 2002). In our study, DM animals treated with L-NAME had no compensatory increase in AQP2 expression. Nitric oxide synthase inhibition by L-NAME has been shown to inhibit vasopressin release (Mornagui et al., 2010), perhaps explaining the lowered levels of AQP2 expression the treated DM animals.

In our studies, L-NAME treatment had no effect on NKCC2 expression. These findings do not corroborate with other studies which observed an increase of NKCC2 abundance in response to
L-NAME (Kim et al., 2006; Wangensteen et al., 2006; Riazi et al., 2009). These studies differ from ours in that the rats were subjected to L-NAME for 4-8 weeks whereas the rats in our study were treated with L-NAME for 3 weeks. In addition, we used a lower concentration of L-NAME during treatment compared to the other studies. While these may be minor factors, alterations in NKCC2 levels have been shown to be time dependent in other animal models (Kim et al., 2003). NKCC2 protein abundance was upregulated in the OM of DM rats. Increased NKCC2 expression likely increases sodium reabsorption and, through countercurrent multiplication, increases urine concentration. L-NAME did not alter the elevated expression of NKCC2 in DM animals. Given the likelihood that treatments of L-NAME longer than 3 weeks increases NKCC2 expression, it would be interesting to see if longer treatment periods amplifies the DM-induced compensatory upregulation of the transporter. NO stimulates production of cGMP which has been shown to decrease surface NKCC2 levels thus rendering the transporter inactive (Ares et al., 2008). It is possible that the L-NAME used in our studies prevents the synthesis of cGMP allowing NKCC2 to accumulate at the plasma membrane where it is active in the DM animals contributing to the reduction of polyuria in the L-NAME-treated DM rats. 

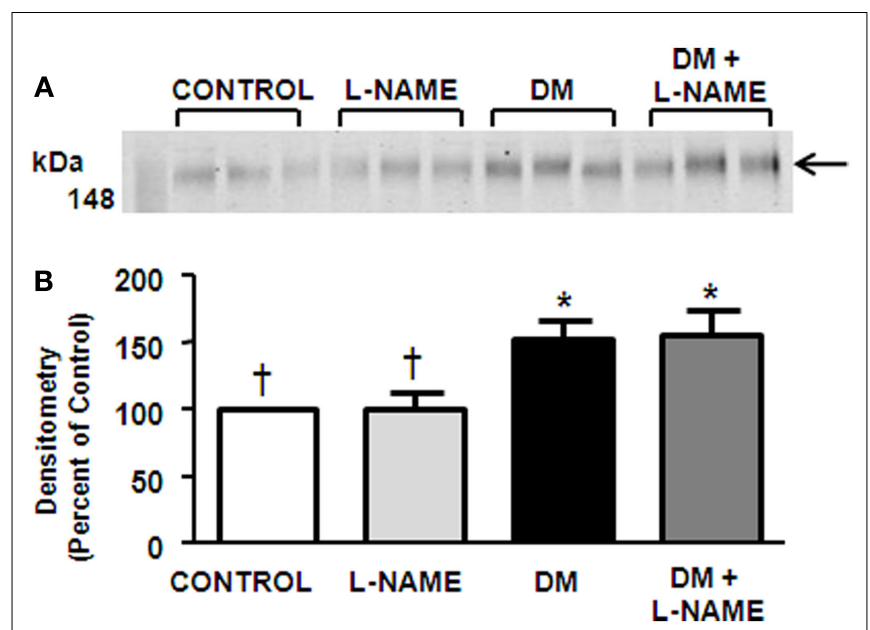

FIGURE 4 | NO inhibition does not affect NKCC2 expression. Observed is a representative western blot of outer medulla (A) probed for NKCC2. Densitometry was determined (B). The experimental conditions were performed five times $(n=5)$ where there were five animals per experimental group in each cohort. In total, 25 animals per experimental group were analyzed. ${ }^{*} p<0.05$ compared to control, ${ }^{\dagger} p<0.05$ compared to DM.

This hypothesis would need to be confirmed with localization experiments.

Long-term treatment of rats with L-NAME can reduce medullary blood flow resulting in a major effect on sodium and water homeostasis and promoting the development of hypertension in these animals (Cowley et al., 1995). We did not measure blood pressure in our experimental animal groups however, it is probable that L-NAME-treated animals were hypertensive. Thus, L-NAME-induced hypertension and not NOS inhibition may be the explanation for the attenuated expression of the concentrated transporters in L-NAME-treated DM animals. Protein abundance of these transporters has been examined in other animal models of hypertension (Klein et al., 2006). In these models UT-A1, AQP2, and NKCC2 were all downregulated. In our L-NAMEtreated animals, which should mimic these hypertension models, we did not see a change in transporter expression levels. Although the contribution of hypertension cannot be ruled out, because the expected decrease of UT-A1, AQP2, and NKCC2 was not observed, we assume that a majority of the L-NAME effects in our study is due to NOS inhibition and not hypertension.

Several reports have shown that administration of L-NAME to STZ-treated rats reduces the renal hyperfiltration that occurs with uncontrolled diabetes (Ito et al., 2001; Brands et al., 2004). Although it seems plausible that urine flow rates may contribute to urea transporter and AQP2 expression, studies have found that protein levels of these transporters are not responsive to increased urine flow rate or loss of medullary hypertonicity (Marples et al., 1998). For instance, animals with lithium-induced nephrogenic diabetes insipidus produce a large 24 -h urine volume but have reduced $A Q P 2$, UT-A1, and UT-A3 expression rather than the increased expression observed in the DM model (Blount et al., 2010). Thus, we believe that the reduction of urine output in
L-NAME-treated rats is not the explanation for the decrease in transporter expression.

Interpretation of our findings are complicated by conflicting reports of the roles of NO and NOS in the diabetic kidney. Collectively, studies indicate that expression of all isoforms of NOS is increased in the IM of STZ-induced diabetes although by possibly different mechanisms (Lee et al., 2005). Increased NOS1 expression was found to be due to high blood glucose but not glycosuria whereas increased NOS3 activity in the IMCD is attributed to sheer stress stimuli due to the high urine flow associated with diabetes (Lee et al., 2005). Despite reports of localized increases of NO production in the IMCD (Choi et al., 1999), we observed a decrease in NO in the urine of diabetic rats. Since urinary NOx is an indicator of total NO production in an animal (Wang et al., 2011), we can conclude that the reported increase in NOS activity in the IMCD is not sufficient to prevent an overall decrease in total body NO production. NO concentration in the kidney changes with advancing diabetes (Komers and Anderson, 2003). In early diabetes, there is an excess of NO which likely contributes to renal hyperfiltration and hyperperfusion. Hyperfiltration was shown to be reversed via L-NAME administration to STZ-injected rats (Levin-Iaina et al., 2011). Advanced stages of diabetes lower NO levels in the kidney which contribute to the declining renal function. Urinary nitrite/nitrate excretion is decreased in rodent models of chronic diabetes (Trachtman et al., 2002) suggesting that the animal model used in these studies resembles later stages of diabetes.

The initial increase of NOS isoforms in diabetes may further complicate disease progression. Each isoform of NOS requires five cofactors/prosthetics to produce NO from L-arginine. Reduction of these cofactors and/or substrate, frequently observed in advanced diabetes, leads to the uncoupling of NOS resulting in the synthesis of $\mathrm{O}_{2}^{-}$instead of $\mathrm{NO}$ (Forbes et al., 2008). Increased concentrations of $\mathrm{O}_{2}^{-}$in diabetic animals could explain the increased expression of the urea transporters in the diabetic IM given that urea permeability is increased by $\mathrm{O}_{2}^{-}$(Zimpelmann et al., 2003). In our studies, L-NAME may be exerting the observed effect by reducing the elevated $\mathrm{O}_{2}^{-}$levels in diabetic animals. Although in some reports, administration of L-NAME significantly increased $\mathrm{O}_{2}^{-}$levels (Usui et al., 1999; Heiman and Allen-Gipson, 2000). It would therefore be interesting to examine the fate of the concentrating transporters in diabetic animals that were treated with L-NAME and proven $\mathrm{O}_{2}^{-}$production inhibitors such as allopurinol, ebselen, and NAC.

Reports indicate that diabetes affects the individual isoforms of NOS differently. Diabetic mice lacking NOS3 do not have severe oxidative stress and tubulointerstitial fibrosis compared to control, diabetic mice (Wang et al., 2011). However, diabetic nephropathy was more severe in STZ-induced diabetic NOS2 knockout mice than STZ-injected wild type mice (Trachtman et al., 2002). It has been suggested that cellular distribution of NOS isoforms may explain conflicting effects. In the IM, NOS1 is largely cytosolic while NOS2 and NOS3 levels are more associated with membrane enriched fractions (Lee et al., 2005). Cytosolic NOS activity, due to NOS3, is increased earlyonset diabetes (Lee et al., 2005). L-NAME inhibits all isoforms of NOS thus we were unfortunately not able to address the 
impact that individual NOS isoforms have on diabetes-induced polyuria.

In conclusion, we found that inhibition of NOS activity can reduce the advancing polyuria associated with diabetes. Uncontrolled diabetes increased total protein abundance of the urine concentrating transporters, UT-A1, UT-A3, and AQP2 by increasing glyco-forms of the transporters in a compensatory manner. While not altering the enhanced glycosylation state of these

\section{REFERENCES}

Albertoni Borghese, M. F., Majowicz, M. P., Ortiz, M. C., Delgado, M. F., Sterin Speziale, N. B., and Vidal, N. A. (2007). Renal sodiumglucose cotransporter activity and aquaporin-2 expression in rat kidney during chronic nitric oxide synthase inhibition. Nephron Physiol. 107, p77-p86.

Ares, G. R., Caceres, P., AlvarezLeefmans, F. J., and Ortiz, P. A. (2008). cGMP decreases surface NKCC2 levels in the thick ascending limb: role of phosphodiesterase 2 (PDE2). Am. J. Physiol. Renal Physiol. 295, F877-F887.

Bardoux, P., Martin, H., Ahloulay, M., Schmitt, F., Bouby, N., Trinh-Trang-Tan, M. M., and Bankir, L. (1999). Vasopressin contributes to hyperfiltration, albuminuria, and renal hypertrophy in diabetes mellitus: study in vasopressin-deficient Brattleboro rats. Proc. Natl. Acad. Sci. U.S.A. 96, 10397-10402.

Blount, M. A., Sands, J. M., Kent, K. J., Smith, T. D., Price, S. R., and Klein, J. D. (2008). Candesartan augments compensatory changes in medullary transport proteins in the diabetic rat kidney. Am. J. Physiol. Renal Physiol. 294, F1448-F1452.

Blount, M. A., Sim, J. H., Zhou, R., Martin, C. F., Lu, W., Sands, J. M., and Klein, J. D. (2010). Expression of transporters involved in urine concentration recovers differently after cessation of lithium treatment. Am. J. Physiol. Renal Physiol. 298, F601-F608.

Bouley, R., Pastor-Soler, N., Cohen, O., Mclaughlin, M., Breton, S., and Brown, D. (2005). Stimulation of AQP2 membrane insertion in renal epithelial cells in vitro and in vivo by the cGMP phosphodiesterase inhibitor sildenafil citrate (Viagra). Am. J. Physiol. Renal Physiol. 288, F1103-F1112.

Brands, M. W., Bell, T. D., and Gibson, B. (2004). Nitric oxide may prevent hypertension early in diabetes by counteracting renal actions of superoxide. Hypertension 43, 57-63.
Centers for Disease Control and Prevention. (2008). National Diabetes Fact Sheet: General Information and National Estimates on Diabetes in the United States, 2007. Atlanta, GA: Department of Health and Human Services, Centers for Disease Control and Prevention.

Chen, G., Howe, A. G., Xu, G., Frohlich, O., Klein, J. D., and Sands, J. M. (2011). Mature N-linked glycans facilitate UT-A1 urea transporter lipid raft compartmentalization. FASEB J. 25, 4531-4539.

Choi, K. C., Lee, S. C., Kim, S. W., Kim, N. H., Lee, J. U., and Kang, Y. J. (1999). Role of nitric oxide in the pathogenesis of diabetic nephropathy in streptozotocin-induced diabetic rats. Korean J. Intern. Med. 14, 32-41.

Cowley, A. W. Jr., Mattson, D. L., Lu, S., and Roman, R. J. (1995). The renal medulla and hypertension. Hypertension 25, 663-673.

Forbes, J. M., Coughlan, M. T., and Cooper, M. E. (2008). Oxidative stress as a major culprit in kidney disease in diabetes. Diabetes 57, 1446-1454.

He, Y., Fan, Z., Zhang, J., Zhang, Q., Zheng, M., Li, Y., Zhang, D., Gu, S., and Yang, H. (2011). Polymorphisms of eNOS gene are associated with diabetic nephropathy: a meta-analysis. Mutagenesis 26, 339-349.

Heiman, A. S., and Allen-Gipson, D. (2000). Cytokines potentiate human eosinophil superoxide generation in the presence of $\mathrm{N}$ (omega)-nitro-L-arginine methyl ester. Int. J. Immunopharmacol. 22, 171-181.

Herrera, M., Hong, N. J., Ortiz, P. A., and Garvin, J. L. (2009). Endothelin1 inhibits thick ascending limb transport via Akt-stimulated nitric 1454-1460.

Huang, J. S., Chuang, L. Y., Guh, J. Y., and Huang, Y. J. (2009). Effects of nitric oxide and antioxidants on advanced glycation end productsinduced hypertrophic growth in human renal tubular cells. Toxicol. Sci. 111, 109-119. oxide production. J. Biol. Chem. 284, transporters, inhibition of NO in DM prevented the increased total expression of these transporters.

\section{ACKNOWLEDGMENTS}

Work was supported by the following NIH/NIDDK grants: T32 DK007656 (Penelope Cipriani), STEP-UP (Sunhye L. Kim), K01-DK082733 (Mitsi A. Blount), K01-DK082733-S1 (Mitsi A. Blount).

Ito, A., Uriu, K., Inada, Y., Qie, Y. L., Takagi, I., Ikeda, M., Hashimoto, O., Suzuka, K., Eto, S., Tanaka, Y., and Kaizu, K. (2001). Inhibition of neuronal nitric oxide synthase ameliorates renal hyperfiltration in streptozotocin-induced diabetic rat. J. Lab. Clin. Med. 138, 177-185.

Iwasaki, Y., Kondo, K., Murase, T., Hasegawa, H., and Oiso, Y. (1996). Osmoregulation of plasma vasopressin in diabetes mellitus with sustained hyperglycemia. J. Neuroendocrinol. 8, 755-760.

Kim, D., Klein, J. D., Racine, S., Murrell, B. P., and Sands, J. M. (2005). Urea may regulate urea transporter protein abundance during osmotic diuresis. Am. J. Physiol. Renal Physiol. 288, F188-F197.

Kim, D., Sands, J. M., and Klein, J. D. (2003). Changes in renal medullary transport proteins during uncontrolled diabetes mellitus in rats. Am. J. Physiol. Renal Physiol. 285, F303F309.

Kim, J. S., Choi, K. C., Jeong, M. H., Kim, S. W., Oh, Y. W., and Lee, J. U. (2006). Increased expression of sodium transporters in rats chronically inhibited of nitric oxide synthesis. J. Korean Med. Sci. 21, 1-4.

Klein, J. D., Murrell, B. P., Tucker, S., Kim, Y. H., and Sands, J. M. (2006). Urea transporter UT-A1 and aquaporin-2 proteins decrease in response to angiotensin II or norepinephrine-induced acute hypertension. Am. J. Physiol. Renal Physiol. 291, F952-F959.

Komers, R., and Anderson, S. (2003). Paradoxes of nitric oxide in the diabetic kidney. Am. J. Physiol. Renal Physiol. 284, F1121-F1137.

Lee, D. L., Sasser, J. M., Hobbs, J. L., Boriskie, A., Pollock, D. M., Carmines, P. K., and Pollock, J. S. (2005). Posttranslational regulation of NO synthase activity in the renal medulla of diabetic rats. Am. J. Physiol. Renal Physiol. 288, F82-F90.

Levin-Iaina, N., Iaina, A., and Raz, I. (2011). The emerging role of NO and IGF-1 in early renal hypertrophy in STZ-induced diabetic rats. Diabetes Metab. Res. Rev. 27, 235-243.
Marples, D., Christensen, B. M., Frokiaer, J., Knepper, M. A., and Nielsen, S. (1998). Dehydration reverses vasopressin antagonist-induced diuresis and aquaporin-2 downregulation in rats. Am. J. Physiol. 275, F400-F409.

Martin, A., Rojas, S., Chamorro, A., Falcon, C., Bargallo, N., and Planas, A. M. (2006). Why does acute hyperglycemia worsen the outcome of transient focal cerebral ischemia? Role of corticosteroids, inflammation, and protein O-glycosylation. Stroke 37, 1288-1295.

Morishita, T., Tsutsui, M., Shimokawa, H., Sabanai, K., Tasaki, H., Suda, O., Nakata, S., Tanimoto, A., Wang, K.Y., Ueta, Y., Sasaguri, Y., Nakashima, Y., and Yanagihara, N. (2005) Nephrogenic diabetes insipidus in mice lacking all nitric oxide synthase isoforms. Proc. Natl. Acad. Sci. U.S.A. 102, 10616-10621.

Mornagui, B., Rezg, R., Grissa, A., Duvareille, M., Gharib, C., Kamoun, A., El-Fazaa, S., and Gharbi, N. (2010). Influence of nitric oxide synthase inhibition on vasopressin and corticosterone secretion during water deprivation in rats. J. Physiol. Biochem. 66, 271-281.

Nejsum, L. N., Kwon, T. H., Marples, D., Flyvbjerg, A., Knepper, M. A. Frokiaer, J., and Nielsen, S. (2001). AQP2, and AQP3 expression in rats with diabetes mellitus. Am. J. Physiol. Renal Physiol. 280, F715-F726.

Nielsen, S., Frokiaer, J., Marples, D., Kwon, T. H., Agre, P., and Knepper, M. A. (2002). Aquaporins in the kidney: from molecules to medicine. Physiol. Rev. 82, 205-244.

Nonoguchi, H., Sands, J. M., and Knepper, M. A. (1988). Atrial natriuretic factor inhibits vasopressinstimulated osmotic water permeability in rat inner medullary collecting duct. J. Clin. Invest. 82, 1383-1390.

Palm, F., Buerk, D. G., Carlsson, P. O., Hansell, P., and Liss, P. (2005). Reduced nitric oxide concentration in the renal cortex of streptozotocininduced diabetic rats: effects on renal oxygenation and microcirculation. Diabetes 54, 3282-3287. Compensatory increase in AQP2, p- 
Riazi, S., Tiwari, S., Sharma, N., Rash, A., and Ecelbarger, C. M. (2009). Abundance of the $\mathrm{Na}-\mathrm{K}-2 \mathrm{Cl}$ cotransporter NKCC2 is increased by high-fat feeding in Fischer $344 \mathrm{X}$ Brown Norway (F1) rats. Am. J. Physiol. Renal Physiol. 296, F762-F770.

Satake, M., Ikarashi, N., Kagami, M., Ogiue, N., Toda, T., Kobayashi, Y., Ochiai, W., and Sugiyama, K. (2010). Increases in the expression levels of aquaporin- 2 and aquaporin- 3 in the renal collecting tubules alleviate dehydration associated with polyuria in diabetes mellitus. Biol. Pharm. Bull. 33, 1965-1970.

Trachtman, H., Futterweit, S., Pine, E., Mann, J., and Valderrama, E. (2002). Chronic diabetic nephropathy: role of inducible nitric oxide synthase. Pediatr. Nephrol. 17, 20-29.

Trinder, D., Phillips, P. A., Stephenson, J. M., Risvanis, J., Aminian, A., Adam, W., Cooper, M., and Johnston, C. I. (1994). Vasopressin V1 and V2 receptors in diabetes mellitus. Am. J. Physiol. 266, E217-E223.

Usui, M., Egashira, K., Kitamoto, S., Koyanagi, M., Katoh, M., Kataoka, C., Shimokawa, H., and Takeshita, A. (1999). Pathogenic role of oxidative stress in vascular angiotensinconverting enzyme activation in long-term blockade of nitric oxide synthesis in rats. Hypertension 34, 546-551.

Wang, C. H., Li, F., Hiller, S., Kim, H. S., Maeda, N., Smithies, O., and Takahashi, N. (2011). A modest decrease in endothelial NOS in mice comparable to that associated with human NOS3 variants exacerbates diabetic nephropathy. Proc. Natl. Acad. Sci. U.S.A. 108, 2070-2075.

Wangensteen, R., Rodriguez-Gomez, I., Moreno, J. M., Vargas, F., and Alvarez-Guerra, M. (2006). Chronic nitric oxide blockade modulates renal $\mathrm{Na}-\mathrm{K}-2 \mathrm{Cl}$ cotransporters. J. Hypertens. 24, 2451-2458.
Wu, F., Park, F., Cowley, A. W. Jr., and Mattson, D. L. (1999). Quantification of nitric oxide synthase activity in microdissected segments of the rat kidney. Am. J. Physiol. 276, F874-F881.

Zhao, H. J., Wang, S., Cheng, H., Zhang, M. Z., Takahashi, T., Fogo, A. B., Breyer, M. D., and Harris, R. C. (2006). Endothelial nitric oxide synthase deficiency produces accelerated nephropathy in diabetic mice. J. Am. Soc. Nephrol. 17, 2664-2669.

Zimpelmann, J., Li, N., and Burns, K. D. (2003). Nitric oxide inhibits superoxide-stimulated urea permeability in the rat inner medullary collecting duct. Am. J. Physiol. Renal Physiol. 285, F1160-F1167.

Conflict of Interest Statement: The authors declare that the research was conducted in the absence of any commercial or financial relationships that could be construed as a potential conflict of interest.

Received: 30 January 2012; accepted: 14 May 2012; published online: 06 June 2012.

Citation: Cipriani P, Kim SL, Klein $J D$, Sim JH, von Bergen $T N$ and Blount MA (2012) The role of nitric oxide in the dysregulation of the urine concentration mechanism in diabetes mellitus. Front. Physio. 3:176. doi: 10.3389/fphys.2012.00176

This article was submitted to Frontiers in Renal and Epithelial Physiology, a specialty of Frontiers in Physiology. Copyright (c) 2012 Cipriani, Kim, Klein, Sim, von Bergen and Blount. This is an open-access article distributed under the terms of the Creative Commons Attribution Non Commercial License, which permits non-commercial use, distribution, and reproduction in other forums, provided the original authors and source are credited. 